\title{
Association of HLA class I and II gene polymorphisms with acetaminophen-related Stevens-Johnson syndrome with severe ocular complications in Japanese individuals
}

\author{
Mayumi Ueta ${ }^{1}$, Ryosuke Nakamura², Yoshiro Saito ${ }^{2}$, Katsushi Tokunaga ${ }^{3,8}$, Chie Sotozono ${ }^{4}$, Toshio Yabe ${ }^{5}$, \\ Michiko Aihara ${ }^{6}, K^{2}$ ayoko Matsunaga ${ }^{7}$ and Shigeru Kinoshita ${ }^{1}$
}

\section{Abstract}

Stevens-Johnson syndrome (SJS) and toxic epidermal necrolysis (TEN) are acute-onset mucocutaneous diseases induced by infectious agents and/or inciting drugs. We have reported that the main causative drugs for SJS/TEN with severe ocular complications (SOC) were cold medicines, including multi-ingredient cold medications and nonsteroidal anti-inflammatory drugs (NSAIDs). Moreover, we also reported that acetaminophen is the most frequent causative drug in various cold medicines. In this study, we focused on acetaminophen-related SJS/TEN with SOC and analyzed HLA-class II (HLA-DRB1, DQB1) in addition to HLA-class I (HLA-A, B, C). We studied the histocompatibility antigen genes HLA-DRB1 and DQB1 in addition to HLA-A, B, and C in 80 Japanese patients with acetaminophen-related SJS/TEN with SOC. We performed polymerase chain reaction amplification followed by hybridization with sequence-specific oligonucleotide probes (PCR-SSO) using commercial bead-based typing kits. We also used genotyped data from 113 healthy volunteers for HLA-DRB1 and DQB1, and 639 healthy volunteers for HLA-A, B, and C. HLA-DRB1*08:03 and $D R B 1 * 12: 02$ were associated with acetaminophen-related SJS/TEN with SOC, although the results ceased to be significant when we corrected the $p$-value for the number of alleles detected. HLA-A*02:06 was strongly associated with acetaminophen-related SJS/TEN with SOC (carrier frequency: $p=4.7 \times 10^{-12}, \mathrm{PC}=6.6 \times 10^{-11}, \mathrm{OR}=6.0$; gene frequency: $p=8.0 \times 10^{-13}, \mathrm{PC}=1.1 \times 10^{-11}, \mathrm{OR}=4.9$ ). HLA-B*13:01 (carrier frequency: $p=2.0 \times 10^{-3}, \mathrm{PC}=0.042, \mathrm{OR}=$ 4.1; gene frequency: $p=2.2 \times 10^{-3}, \mathrm{PC}=0.047, \mathrm{OR}=3.9$ ), $H L A-B^{*} 44: 03$ (carrier frequency: $p=2.1 \times 10^{-3}, \mathrm{PC}=0.045$, $\mathrm{OR}=2.4$ ) and $H L A-C^{*} 14: 03$ (carrier frequency: $p=3.4 \times 10^{-3}, \mathrm{PC}=0.045, \mathrm{OR}=2.3$ ) were also significantly associated, while HLA-A*24:02 was inversely associated (gene frequency: $p=6.3 \times 10^{-4}, \mathrm{PC}=8.8 \times 10^{-3}, \mathrm{OR}=0.5$ ).

Acetaminophen-related SJS/TEN with SOC was not associated with HLA-class I/ (HLA-DRB1, DQB1). However, for acetaminophen-related SJS/TEN with SOC, we found an association with HLA-B*13:01 and HLA- C*14:03 in addition to $H L A-A^{*} 02: 06$ and $H L A-B^{*} 44: 03$, which have been described previously.
\end{abstract}

Correspondence: Mayumi Ueta (mueta@koto.kpu-m.ac.jp)

${ }^{1}$ Department of Frontier Medical Science and Technology for Ophthalmology, Kyoto Prefectural University of Medicine, Kyoto, Japan

${ }^{2}$ Division of Medicinal Safety Science, National Institute of Health Sciences, Kawasaki, Japan

Full list of author information is available at the end of the article

\section{Introduction}

Stevens-Johnson syndrome (SJS) is an acute inflammatory vesiculobullous reaction of the skin and the mucosa of the ocular surface, oral cavity, and genitals. Patients with extensive skin detachment and a poor prognosis have toxic epidermal necrolysis (TEN).

In the acute stage of SJS/TEN, approximately $50 \%$ of patients present with severe ocular lesions, such as severe

\section{(c) The Author(s) 2019}

(c) (i) Open Access This article is licensed under a Creative Commons Attribution 4.0 International License, which permits use, sharing, adaptation, distribution and reproduction c. in any medium or format, as long as you give appropriate credit to the original author(s) and the source, provide a link to the Creative Commons license, and indicate if changes were made. The images or other third party material in this article are included in the article's Creative Commons license, unless indicated otherwise in a credit line to the material. If material is not included in the article's Creative Commons license and your intended use is not permitted by statutory regulation or exceeds the permitted use, you will need to obtain permission directly from the copyright holder. To view a copy of this license, visit http://creativecommons.org/licenses/by/4.0/. 
conjunctivitis with pseudomembrane and ocular surface epithelial defects ${ }^{1}$.

The mortality rate of SJS/TEN is high (3\% for SJS and $27 \%$ for TEN $)^{2}$, although its reported annual incidence is only 1-6 per million individuals ${ }^{1}$. The extreme rarity of cutaneous and ocular surface reactions to drug therapies led us to suspect individual susceptibility.

While associations between SJS/TEN and many kinds of inciting drugs have been documented ${ }^{3}$, we have reported that the main causative drugs for SJS/TEN with severe ocular complications (SOC) were cold medicines; approximately $80 \%$ of our SJS/TEN with SOC patients developed SJS/TEN within several days after taking cold medicines, including multi-ingredient cold medications and nonsteroidal anti-inflammatory drugs (NSAIDs), to combat the common cold ${ }^{4-7}$. Moreover, we also reported that acetaminophen is the most frequent causative drug ingredient in various cold medicines and that cold medicine-related SJS/TEN with SOC, including acetaminophen-related SJS/TEN with SOC, was significantly associated with $H L A-A * 02: 06$ and $H L A-B * 44: 03$ in Japanese individuals ${ }^{6}$. However, in a previous study in which we focused on cold medicines, we analyzed only HLA-class I (HLA-A, B, and C).

On the other hand, Power et al. ${ }^{8}$ reported that HLA$D Q B 1^{*} 06: 01$ was associated with Caucasian patients with ocular complications of SJS.

In this study, we focused on acetaminophen-related SJS/ TEN with SOC and analyzed HLA-class II (HLA-DRB1, $D Q B 1)$ in addition to HLA-class I (HLA-A, B, C).

\section{Materials and methods Patients}

Our study was approved by the institutional review boards of Kyoto Prefectural University of Medicine, Kyoto, Japan, and the National Institute of Health Sciences, Kawasaki, Japan. All experimental procedures were conducted in accordance with the principles set forth in the Helsinki Declaration and Ethical Guidelines for Human Genome/Gene Analysis Research of Japan. The purpose of the study and the experimental protocols were explained to all participants, and their prior written informed consent was obtained.

Because ophthalmologists encounter patients not only in the acute stage but also in the chronic stage, it is not easy for ophthalmologists to render a differential diagnosis of SJS or TEN when patients present in the chronic stage because the vesiculobullous skin lesions evident in the acute stage have healed by the chronic stage ${ }^{5}$. Thus, our ophthalmologic diagnosis of SJS/TEN was based on a confirmed history of acute-onset high fever, serious mucocutaneous illness with skin eruptions, and the involvement of at least 2 mucosal sites, including the ocular surface $e^{4,6,7,9,10}$.
SJS/TEN patients with SOC in the acute stage often suffer severe ocular sequelae such as vision loss and very severe dry eye that prevent them from having a normal life $^{11}$. We defined acute-stage SOC as a condition with severe conjunctivitis with pseudomembrane and epithelial defects on the ocular surface (cornea and/or conjunctiva $)^{12}$ and chronic-stage SOC as a condition with ocular sequelae such as severe dry eye, trichiasis, symblepharon, and conjunctival invasion into the cornea ${ }^{11}$.

For HLA genotyping, we enrolled 80 Japanese SJS/ TEN with SOC patients (64 of them were recruited by Kyoto Prefectural University of Medicine, and 16 of them were recruited by the Japan Severe Adverse Reactions research group (JSAR research group, mainly operated by the National Institute of Health Sciences)). The average patient age was $38.9 \pm 17.6$ (SD) years, and the average onset age was $30.1 \pm 16.5(\mathrm{SD})$ years; the male:female ratio was 32:48. Note that the results of HLA class I (HLA-A, B, and C types) from 73 cases of acetaminophen-related SJSTEN patients with SOC were reported previously ${ }^{6}$.

\section{Controls}

We used the genotyped data of 113 healthy volunteer blood donors for HLA class II (DRB1, DQB1) and of 639 healthy volunteers for $H L A$ class $I(A, B, C)$. All volunteers were Japanese residing in Japan. They have been used in our previous study ${ }^{6,9}$.

\section{HLA genotyping}

We studied the histocompatibility antigen genes $H L A$ $A, B, C, D R B 1$, and $D Q B 1$ of 80 Japanese acetaminophenrelated SJS/TEN with SOC patients. These alleles were detected by the PCR-Luminex typing method using the WAKFlow HLA Typing Kit (Wakunaga, Hiroshima) as previously reported ${ }^{6,9,13}$. Genotype determination and data analysis were performed automatically using the WAKFlow typing software.

\section{Statistical methods}

We compared the carrier frequency of individual $H L A$ alleles in our patients and controls based on the dominant model using Fisher's exact test (JMP version 11 software; SAS Institute Japan Ltd., Tokyo, Japan). Each allele was assessed as an independent variable, and separate $p$-values were calculated. A $p$-value of $<0.05$ was regarded as significant. In addition, the $p$-values were corrected for the number of alleles tested. The alleles with total numbers under 8 for HLA-class II (DRB1, DQB1) and under 21 for $H L A$ class $I(A, B, C)$ were included in others.

\section{Results}

Table 1 shows the results on HLA-DRB1 alleles. Although the correction of the $p$-values for the number of 
alleles detected $(n=16)$ rendered the result not significant, HLA-DRB1*08:03 (carrier frequency: $p=0.016$, $\mathrm{Pc}=0.26, \mathrm{OR}=2.5$; gene frequency: $p=0.017, \mathrm{Pc}=0.17$, $\mathrm{OR}=2.3$ ) and $D R B 1 * 12: 02$ (carrier frequency: $p=0.024$, $\mathrm{Pc}=0.38, \mathrm{OR}=3.9$; gene frequency: $p=0.027, \mathrm{Pc}=0.27$, $\mathrm{OR}=3.7)$ were associated with acetaminophen-related SJS/TEN with SOC. There was also no association between $H L A-D Q B 1$ and acetaminophen-related SJS/TEN with SOC (Supplementary Table 1 ).

As shown in Table 2a, HLA-A*02:06 was strongly associated with acetaminophen-related SJS/TEN with SOC (carrier frequency: $p=4.7 \times 10^{-12}, \mathrm{Pc}=6.6 \times 10^{-11}$, $\mathrm{OR}=6.0 ;$ gene frequency: $p=8.0 \times 10^{-13}, \quad \mathrm{Pc}=1.1 \times$ $\left.10^{-11}, \mathrm{OR}=4.9\right) . H L A-A * 24: 02$ was inversely associated (carrier frequency: $p=5.3 \times 10^{-3}, \mathrm{Pc}=0.074, \mathrm{OR}=0.5$; gene frequency: $p=6.3 \times 10^{-4}, \mathrm{Pc}=8.8 \times 10^{-3}, \mathrm{OR}=$ 0.5). HLA-A*33:03 was also associated (carrier frequency: $p=0.011, \mathrm{Pc}=0.16$, OR $=2.1$; gene frequency: $p=0.12$, $\mathrm{Pc}=0.16, \quad \mathrm{OR}=2.0$ ), and $H L A-A * 11: 01$ (carrier frequency: $p=0.024, \mathrm{Pc}=0.34, \mathrm{OR}=0.4$; gene frequency: $p=0.037, \mathrm{Pc}=0.52, \mathrm{OR}=0.4$ ) and $A^{*} 26: 01$ (gene frequency: $p=0.047, \mathrm{Pc}=0.65, \mathrm{OR}=0.4$ ) were inversely associated, but the association ceased to be significant when we corrected the $p$-value for the number of alleles detected $(n=14)$.

Table $2 \mathrm{~b}$ shows the results on HLA-B alleles. $B * 13: 01$ (carrier frequency: $p=2.0 \times 10^{-3}, \mathrm{Pc}=0.042, \mathrm{OR}=4.1$; gene frequency: $p=2.2 \times 10^{-3}, \mathrm{Pc}=0.047, \mathrm{OR}=3.9$ ), and $H L A-B^{*} 44: 03$ (carrier frequency: $p=2.1 \times 10^{-3}, \quad \mathrm{PC}=$ $0.045, \mathrm{OR}=2.4$; gene frequency: $p=3.7 \times 10^{-3}, \mathrm{Pc}=$ 0.078 , OR $=2.1)$ was significantly associated. $H L A$ $B * 46: 01$ was also associated (carrier frequency: $p=0.025$, $\mathrm{Pc}=0.53, \mathrm{OR}=2.2$; gene frequency: $p=0.030, \mathrm{Pc}=0.64$, $\mathrm{OR}=2.1$ ), and $H L A-B^{*} 15: 01$ (carrier frequency: $p=4.8 \times$ $10^{-3}, \mathrm{Pc}=0.10, \mathrm{OR}=0.3$; gene frequency: $p=4.7 \times 10^{-3}$, $\mathrm{Pc}=0.98, \mathrm{OR}=0.3$ ) and $B * 52: 01$ (carrier frequency: $p=$ $0.014, \mathrm{Pc}=0.30, \mathrm{OR}=0.4$; gene frequency: $p=0.021$, $\mathrm{Pc}=0.43, \mathrm{OR}=0.4$ ) were inversely associated, although the results ceased to be significant when we corrected the $p$-value for the number of alleles detected $(n=21)$.

Table 2c shows the results for HLA-C alleles. HLA$C^{*} 14: 03$ (carrier frequency: $p=3.4 \times 10^{-3}, \mathrm{Pc}=0.045$, $\mathrm{OR}=2.3$; gene frequency: $p=6.3 \times 10^{-3}, \mathrm{Pc}=0.083$, OR $=2.0$ ) was significantly associated. $H L A-C * 03: 04$ was also associated (carrier frequency: $p=0.048, \mathrm{Pc}=0.63, \mathrm{OR}=$ 1.7), and $H L A-C^{*} 12: 02$ was inversely associated (carrier frequency: $p=0.014, \mathrm{Pc}=0.19, \mathrm{OR}=0.4$; gene frequency: $p=0.021, \mathrm{Pc}=0.27, \mathrm{OR}=0.4$ ), although the results ceased to be significant when we corrected the $p$-value for the number of alleles detected $(n=13)$. The results of $H L A$-class I (HLA-A, B, C) are shown in Supplementary Table 2. 


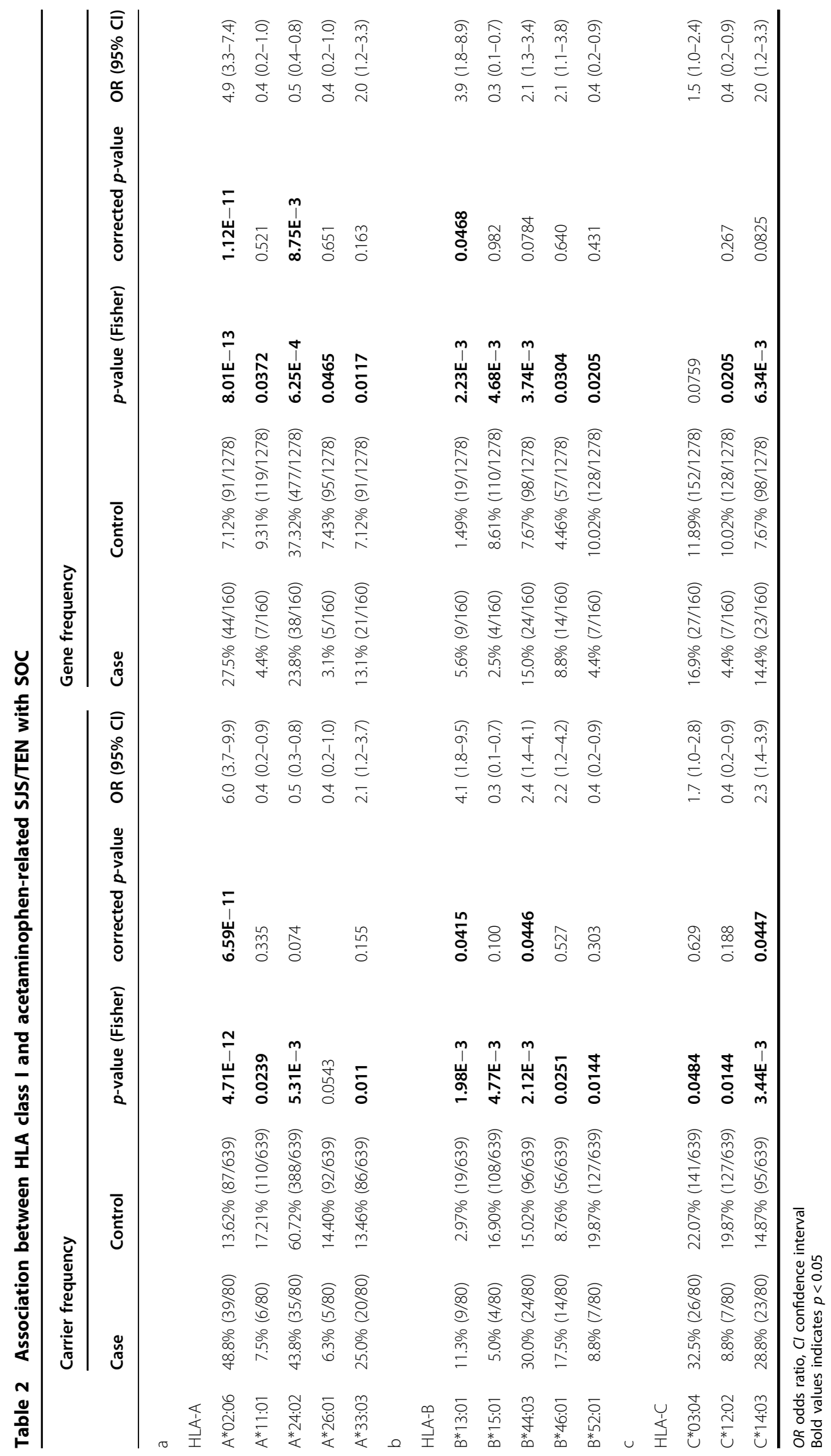




\section{Discussion}

We previously reported that approximately $80 \%$ of our SJS/TEN with SOC patients developed SIS/TEN within several days after taking cold medicines, including multiingredient cold medications and NSAIDs, to combat the common $\operatorname{cold}^{4,5,7,13}$. More than half of SIS/TEN with SOC patients developed SJS after taking cold medicines in the Brazilian population ${ }^{14}$. Cold medicine, including NSAIDs, might be associated with SOC in SJS/TEN patients in the Korean population ${ }^{15}$. Moreover, $69 \%$ of SJS/TEN with SOC patients had a history of taking cold medicine before the onset of SJS/TEN in the Thai population ${ }^{16}$.

Acetaminophen, also called paracetamol, is the most frequent causative drug for cold medicine-related SIS/ TEN with SOC in Japanese ${ }^{6}$ and Thai individuals ${ }^{16}$. On the other hand, dipyrone is the most frequent cause of cold medicine-related SIS/TEN with SOC in Brazil ${ }^{14}$.

In this study, we analyzed both class I and class II HLA types focused on acetaminophen-related SJS/TEN with SOC and found that there was also no association between HLA class II (HLA-DQB1, DQB1) and acetaminophen-related SJS/TEN with SOC and confirmed the strong association with $H L A-A * 02: 06$ and the significant association with $H L A-B^{*} 44: 03$, as in our previous study ${ }^{6}$. Moreover, we also found associations with $H L A-B * 13: 01$ and $H L A-C^{*} 14: 03$ and an inverse association with $H L A-A * 24: 02$.

Power et al. ${ }^{8}$ reported that $H L A-D Q B 1 * 06: 01$ was associated with Caucasian patients with ocular complications of SJS. However, we could not detect an association between Japanese SJS/TEN and SOC and HLA$D Q B 1 * 06: 01$.

$H L A-A$ *02:06 was significantly associated with Japanese $^{6}$ and Korean ${ }^{17}$ cold medicine-related SJS/TEN with SOC. On the other hand, Brazilian cold medicine-related SJS/TEN with SOC was significantly associated with HLA$A * 66: 01$ in individuals with both Pardo and European ancestry and $H L A-B^{*} 44: 03$ and $H L A-C^{*} 12: 03$ for European ancestry $^{14}$. Moreover, cold medicine-related SJS/ TEN with SOC in Thailand was significantly associated with the HLA-B*44:03-HLA-C*07:01 haplotype, although their main causative drug is paracetamol ${ }^{16}$. Thus, there are ethnic differences in associated HLAs with cold medicine-related SJS/TEN with SOC.

Acetaminophen-associated HLA type might be quite similar to HLA associated with cold medicine-related SIS/TEN with SOC. The binding modes of acetaminophen, ibuprofen, and loxoprofen at the antigenic peptide-binding groove of the HLA-A $* 02: 06$ molecule are different because their molecules are not similar, and their composite risk indexes are also different ${ }^{18}$. Thus, we suggest that the common function of cold medicines, such as acetaminophen and NSAIDs, is most important for the onset of SJS/TEN with SOC. The common function of cold medicines is a suppression function of prostaglandin $\mathrm{E}_{2}\left(\mathrm{PGE}_{2}\right)$ production, which suppresses mucocutaneous inflammation; $\mathrm{PGE}_{2}$ acts at $\mathrm{EP} 3\left(\mathrm{PGE}_{2}\right.$ receptor 3), which is one of the 4 receptors (EP1, EP2, $\mathrm{EP3}, \mathrm{EP} 4)$ for $\mathrm{PGE}_{2}$, in the epidermis ${ }^{19}$ and mucosal epithelium ${ }^{10,20}$, negatively regulating mucocutaneous inflammation. Cold medicine, including acetaminophen, could downregulate inflammatory suppressing mechanism(s) by $\mathrm{PGE}_{2}$ and might augment the abnormal immune response resulting in the induction of SJS/TEN with $\mathrm{SOC}^{5,21}$.

Acetaminophen (paracetamol) is thought to be a safe drug and is widely prescribed for children with cold symptoms or is widely included in commercial cold medicines. However, for SIS/TEN with SOC, acetaminophen could be the main causative drug. Therefore, physicians should prescribe it with knowledge about SJS/ TEN.

In summary, acetaminophen-related SJS/TEN with SOC was not associated with HLA-class II (HLA-DRB1, DQB1), but we found an association with HLA-B*13:01 and HLA- $C * 14: 03$ in addition to HLA-A*02:06 and HLA$B^{*} 44: 03$, which have been described previously.

\section{Acknowledgements}

This work was supported by grants-in-aid from the Ministry of Education, Culture, Sports, Science and Technology of the Japanese government, by the JSPS Core-to-Core Program, A. Advanced Research Networks, and partially supported by grants-in-aid for scientific research from the Japanese Ministry of Health, Labor and Welfare, and a research grant from the Kyoto Foundation for the Promotion of Medical Science and the Intramural Research Fund of Kyoto Prefectural University of Medicine, and supported by AMED under Grant Number JP19mk0101085.

\section{Author details}

${ }^{1}$ Department of Frontier Medical Science and Technology for Ophthalmology, Kyoto Prefectural University of Medicine, Kyoto, Japan. ${ }^{2}$ Division of Medicinal Safety Science, National Institute of Health Sciences, Kawasaki, Japan.

${ }^{3}$ Department of Human Genetics, Graduate School of Medicine, University of Tokyo, Tokyo, Japan. ${ }^{4}$ Department of Ophthalmology, Kyoto Prefectural University of Medicine, Kyoto, Japan. ${ }^{5}$ Tokyo Metropolitan Red Cross Blood Center, Tokyo, Japan. ${ }^{6}$ Department of Environmental Immuno-Dermatology, Yokohama City University Graduate School of Medicine, Yokohama, Japan.

${ }^{7}$ Department of Integrative Medical Science for Allergic Disease, Fujita Health University School of Medicine, Aichi, Japan. ${ }^{8}$ Present address: Genome Medical Science Project (Toyama), National Center for Global Health and Medicine, Tokyo, Japan

\section{Conflict of interest}

The authors declare that they have no conflict of interest.

\section{Publisher's note}

Springer Nature remains neutral with regard to jurisdictional claims in published maps and institutional affiliations.

Supplementary information is available for this paper at https://doi.org/ 10.1038/s41439-019-0082-6.

Received: 1 July 2019 Revised: 10 September 2019 Accepted: 14 September 2019.

Published online: 28 October 2019 


\section{References}

1. Sotozono, C. et al. Predictive factors associated with acute ocular involvement in Stevens-Johnson syndrome and toxic epidermal necrolysis. Am. J. Ophthalmol. 160, 228-237 e2 (2015).

2. Power, W. J., Ghoraishi, M., Merayo-Lloves, J., Neves, R. A. \& Foster, C. S. Analysis of the acute ophthalmic manifestations of the erythema multiforme/StevensJohnson syndrome/toxic epidermal necrolysis disease spectrum. Ophthalmology 102, 1669-1676 (1995).

3. Yamane, Y., Aihara, M. \& Ikezawa, Z. Analysis of Stevens-Johnson syndrome and toxic epidermal necrolysis in Japan from 2000 to 2006. Allergol. Int 56, 419-425 (2007).

4. Ueta, M. et al. Association between prostaglandin E receptor 3 polymorphisms and Stevens-Johnson syndrome identified by means of a genome-wide association study. J. Allergy Clin. Immunol. 126, 1218-1225 e10 (2010).

5. Ueta, M. \& Kinoshita, S. Ocular surface inflammation is regulated by innate immunity. Prog. Retin. Eye Res. 31, 551-575 (2012).

6. Ueta, M. et al. Independent strong association of HLA-A*02:06 and HLA$B^{*} 44: 03$ with cold medicine-related Stevens-Johnson syndrome with severe mucosal involvement. Sci. Rep. 4, 4862 (2014).

7. Ueta, M. et al. IKZF1, a new susceptibility gene for cold medicine-related Stevens-Johnson syndrome/toxic epidermal necrolysis with severe mucosal involvement. J. Allergy Clin. Immunol. 135, 1538-1545 e17 (2015).

8. Power, W. J. et al. HLA typing in patients with ocular manifestations of Stevens-Johnson syndrome. Ophthalmology 103, 1406-1409 (1996).

9. Ueta, M. et al. HLA class I and II gene polymorphisms in Stevens-Johnson syndrome with ocular complications in Japanese. Mol. Vis. 14, 550-555 (2008).

10. Ueta, M., Matsuoka, T., Narumiya, S. \& Kinoshita, S. Prostaglandin E receptor subtype EP3 in conjunctival epithelium regulates late-phase reaction of experimental allergic conjunctivitis. J. Allergy Clin. Immunol. 123, 466-471 (2009).

11. Sotozono, C. et al. New grading system for the evaluation of chronic ocular manifestations in patients with Stevens-Johnson syndrome. Ophthalmology 114, 1294-1302 (2007).
12. Sotozono, C. et al. Diagnosis and treatment of Stevens-Johnson syndrome and toxic epidermal necrolysis with ocular complications. Ophthalmology 116 685-690 (2009).

13. Ueta, M. et al. Trans-ethnic study confirmed independent associations of HLA-A*02:06 and HLA-B*44:03 with cold medicine-related StevensJohnson syndrome with severe ocular surface complications. Sci. Rep. 4 5981 (2014)

14. Wakamatsu, T. H. et al. Human leukocyte antigen class I genes associated with Stevens-Johnson syndrome and severe ocular complications following use of cold medicine in a Brazilian population. JAMA Ophthalmol. 135, 355-360 (2017).

15. Lee, H. K., Yoon, K. C., Seo, K. Y., Ueta, M. \& Kim, M. K. Chronic ocular complications of Stevens-Johnson syndrome associated with causative medications in Korea. J. Allergy Clin. Immunol. Pr. 6, 700-702 e2 (2018).

16. Jongkhajornpong, P. et al. Association between HLA-B*44:03-HLA$C^{*}$ 07:01 haplotype and cold medicine-related Stevens-Johnson syndrome with severe ocular complications in Thailand. Br. J. Ophthalmol. 102, 1303-1307 (2018)

17. Jun, I. et al. Association of human antigen class I genes with cold medicinerelated Stevens-Johnson syndrome with severe ocular complications in a Korean population. Br. J. Ophthalmol. 103, 573-576 (2019).

18. Isogai H. et al. In Silico Risk Assessment of HLA-A*02:06-Associated StevensJohnson Syndrome and Toxic Epidermal Necrolysis Caused by Cold Medicine Ingredients. J Toxicol. 2013, 514068 (2013).

19. Honda, T. et al. Prostaglandin E(2)-EP(3) signaling suppresses skin inflammation in murine contact hypersensitivity. J. Allergy Clin. Immunol. 124, 809-818 e2 (2009).

20. Kunikata, T. et al. Suppression of allergic inflammation by the prostaglandin E receptor subtype EP3. Nat. Immunol. 6, 524-531 (2005).

21. Ueta, M. Results of detailed investigations into Stevens-Johnson syndrome with severe ocular complications. Invest Ophthalmol. Vis. Sci. 59, DES183-DES191 (2018). 